\title{
Simulation Based Exploration of Bacterial \\ Cross Talk Between Spatially Separated \\ Colonies in a Multispecies Biofilm \\ Community
}

\author{
Pavel Zarva and Hermann J. Eberl ${ }^{(凶)}$ \\ Department of Mathematics and Statistics, University of Guelph, \\ Guelph, ON N1G 2W1, Canada \\ $\{$ pzarva, heberl\}@uoguelph.ca
}

\begin{abstract}
We present a simple mesoscopic model for bacterial crosstalk between growing biofilm colonies. The simulation setup mimics a novel microfludic biofilm growth reactor which allows a 2D description. The model is a stiff quasilinear system of diffusion-reaction equations with simultaneously a super-diffusion singularity and a degeneracy (as in the porous medium equation) that leads to the formation of sharp interfaces with finite speed of propagation and gradient blow up. We use a finite volume method with arithmetic flux averaging, and a time adaptive stiff time integrator. We find that signal and nutrient transport between colonies can greatly control and limit biofilm response to induction signals, leading to spatially heterogeneous biofilm behavior.
\end{abstract}

Keywords: Biofilm $\cdot$ Cross talk $\cdot$ Degenerate diffusion $\cdot$ Mathematical model $\cdot$ Reaction-diffusion $\cdot$ Simulation $\cdot$ Super-diffusion

\section{Introduction}

Bacterial biofilms are microbial aggregates on immersed, biotic or abiotic, surfaces or interfaces. Biofilm communities consist usually of several colonies of same or different species. They form wherever environmental conditions permit microbial growth [18]. In the initial (reversible) stage of biofilm formation cells attach to the surface, called substratum in the biofilm literature, and begin the production of extracellular polymeric substances (EPS), a protective layer in which they are themselves embedded and multiply. The colonies grow and eventually may merge into larger assemblages. In these aggregates, diffusion gradients develop that lead to variations in local growth conditions for cells in dependence of their location in the biofilm. Under some circumstances this can lead to the formation of microniches, which allows for example for anaerobic pockets in otherwise aerobic biofilms. Biofilm based processes are engineered for example in wastewater

Supported by Natural Sciences and Engineering Research Council of Canada (NSERC): RGPIN-2019-05003, RTI-2016-00080.

(C) Springer Nature Switzerland AG 2020

V. V. Krzhizhanovskaya et al. (Eds.): ICCS 2020, LNCS 12143, pp. 228-241, 2020.

https://doi.org/10.1007/978-3-030-50436-6_17 
treatment, soil remediation, or also in biofuel production. On the other hand, in a medical or industrial context, biofilm growth is often unwanted and leads to detrimental consequences, such as biofouling and biocorrosion of equipment, public health risks, or bacterial infections [27]. Biofilm borne infections are more difficult to eradicate than other bacterial infections, for example due to the mechanical and chemical protection that the encasing EPS layer offers to the cells.

Quorum sensing is a mechanism that bacteria use to coordinate gene expression in groups. Cells produce a signal (also called autoinducers) at a basal rate. When the signal concentration passes an induction threshold, the cells undergo a change in gene expression [12]. Initially, as the name suggests, this was thought to be a communication mechanism to assess group strength. However, it is understood now that in spatially structured populations and in non completely mixed environments this induction process is also affected by transport processes acting on the signal [16]. For example it was suggested based on theoretical and computational studies that in hydrodynamic environments larger upstream colonies can play a major role in induction of smaller downstream colonies [10], which also has been confirmed experimentally since [17]. In certain biofilms, quorum sensing is used as a stress response mechanism to control virulence factors, such as elastases, pyocyanin, cyanide and exotoxins [1]. It was suggested that biofilm formation itself, i.e. the onset of EPS production, in some species is controlled by a quorum sensing mechanism [2]. Based on computational studies, it was argued [11] that a quorum sensing induction mechanism can explain a switch from an early stages mode of biofilm growth, during which resources are primarily invested in increasing population size, to a mode of growth during which resources are invested in protection by producing EPS.

Cross-talk refers to bacteria of one species or strain responding to signals produced by another one $[3,4]$. In gram negative bacteria $\mathrm{N}$-acyl homoserone lactones (AHL) are an important group of quorum sensing signalling molecules. Although some strains have been reported to produce up to 20 different AHLs [3], the number of known AHLs is limited [24]. Therefore, some signal molecules are used by, or recognised by several strains or species, leading to interspecies communication. This concept of cross-talk challenges the notion that quorum sensing is purely an autoinduction mechanism.

Mathematical models of biofilms have been used since the 1980s, specifically in wastewater engineering. Originally, these were based on the assumption of stratification parallel to the substratum. At the end of the 1990s, after it became appreciated that biofilms are not flat layers many different models for the growth of spatially heterogeneous biofilms were proposed, utilising agent based techniques, cellular automata, or partial differential equations. The models in the latter groups can be subdivided into such that view a biofilm as a mechanical object, and those that consider them a spatially structured population. Experimentally, biofilms have been characterised as both. One model that can be motivated from both angles is a density dependent diffusion reaction framework $[6,20]$. This model framework has been extended to describe several biofilm processes, including some that involve quorum sensing $[7,8,10,11,13-15,26]$. In our current study we will extend this framework to include a simple example of 
bacterial cross-talk. An earlier example of a model of cross talk in biofilms can be found in [24], in which sender and receiver bacteria were in close proximity, in which it was assumed that biofilm colonies do not grow in time, and in which bacteria received nutrients from an agar layer on which they were grown. In our study the focus is on the role of diffusion of signals and nutrients between colonies that are spaced apart. One of the big, unsolved problems in multi-dimensional biofilm modeling is the inherently multi-scale nature of biofilm processes. Often one is interested in mesocopic features, i.e. in processes on the actual colony scale. To simulate such processes, requires a good set of boundary conditions that describe how the domain of simulation (e.g. a small open section of a much larger flow channel) is connected with the greater physical environment. This problem in simulations is in essence the same problem experimental studies suffer from, for example because modern microscopes can only resolve small sections of an experimental reactor. In this paper we introduce a new and simple computational setup, which circumvents this problem, mimicking a new microfluidic growth chamber for biofilm experiments that was recently introduced in [22].

\section{Mathematical Model}

\subsection{Biological Processes and Governing Equations}

We consider a dual species biofilm community consisting of two species of bacteria. Both species compete for a single, shared, growth limiting carbon substrate, which is subject to Fickian diffusion. Substrate uptake follows Monod kinetics, i.e. the uptake rate is proportional to the local substrate concentration when limited, and approximately constant where and when substrate is abundant. Substrate uptake translates into production of new biomass. We also account for cell death at a constant rate. Biofilm colonies do not expand spatially if locally space is available to accommodate newly produced cells. When the maximum physically attainable biofilm density is approached, colonies start spreading. We model this by the density dependent diffusion mechanism that was introduced in [6] and that can be derived both from the viewpoint of a biofilm as a mechanical object and as a spatially structured microbial populations [20]. Experimentally, biofilms have been characterised as both.

We assumed that the bacteria of one species ('sender') produce a signal molecule at a basal rate. If the local signal concentration passes an induction threshold, cells up-regulate and the rate of signal production increases by one order of magnitude. We do not explicitly distinguish between up- and down-regulated cell fractions, but make this distinction implicitly in terms of local signal concentration, and whether it is above or below the induction threshold value. Upregulated cells are assumed to have different growth behavior than down-regulated cells. More specifically, in this exploratory study, we assume that their substrate uptake rate increases, in order to invest resources into protection mechanisms, which we do not model explicitly. The autoinducer signal is transported in the domain by Fickian diffusion and degrades abiotically. The second species ('receiver') responds to signals and upregulates, i.e. increases substrate uptake if the signal surpasses induction threshold, but it does not produce the signal. 
The model is cast in terms of the dependent variables $M_{1}$, volume fraction of sender species; $M_{2}$, volume fraction of receiver species; $C$, concentration of carbon substrate; $S$ concentration of signal. The independent variables are time $t>0$ and location $x \in \Omega \subset \mathbb{R}^{d}$. The model reads

$$
\begin{aligned}
\partial_{t} M_{1} & =\nabla\left[D\left(M_{1}\right) \nabla M_{1}\right]+\mu_{1} f\left(C ; \kappa_{1}\right) M_{1}-\delta_{1} M_{1} \\
\partial_{t} M_{2} & =\nabla\left[D\left(M_{2}\right) \nabla M_{2}\right]+\mu_{2} f\left(C ; \kappa_{2}\right) M_{2}-\delta_{2} M_{2} \\
\partial_{t} C & =D_{C} \Delta C-\sum_{i=1}^{2} \nu_{i}\left(1+\sigma_{i} h\left(S ; \tau_{i}\right) f\left(C ; \kappa_{i}\right) M_{i}\right. \\
\partial_{t} S & =D_{S} \Delta S+f\left(C ; \kappa_{1}\right)\left(\beta h\left(S, \tau_{1}\right)+\alpha\right) M_{1}-\gamma S_{1}
\end{aligned}
$$

In this model the coefficient function $f$ is the Monod function that describes the dependence of the growth process on the substrate concentration $C$. This function saturates $f \approx 1$ if $C \gg \kappa$ and $f$ is proportional to $C$ as $C \ll \kappa$. Function $h$ is a Hill function that describes the induction process, i.e. the transition from a down-regulated state with $h \approx 0$ when $S \ll \tau_{i}$, to an up-regulated state with $h \approx 1$ if $S \gg \tau$. We have

$$
f(C ; \kappa)=\frac{C}{\kappa+C}, \quad h(S ; \tau)=\frac{S^{n}}{\tau^{n}+S^{n}}, \quad n \approx 2.2 \sim 2.5 .
$$

The function $D(M)$ describes spatial movement of biomass in dependence of local density,

$$
D(M)=D_{M} \frac{M^{a}}{(1-M)^{a}}
$$

It combines two nonlinear diffusion effects: (i) for $M \rightarrow 0$ it behaves like $D(M) \sim M^{a}$, i.e we have a porous medium degeneracy with $D(0)=0$ that implies that the biofilm/water interfaces moves with finite speed, and (ii) for $M \rightarrow 1$ it behaves like $D(M) \sim(1-M)^{-a}$, i.e. we have a super-diffusion singularity, which ensures that biomass expands spatially as $M \rightarrow 1$ and ensures $M<1$ even if biomass continues to grow as long as substrates are available. Both effects are necessary to describe biofilm formation. Note that the biomass motility coefficient $D_{M}$ is much smaller than the diffusion coefficients $D_{C, S}$ of substrate and signal. We remark that a single species model formulation is used here for both biomass species independently, although mutli-species formulations (with or without cross-diffusion) are available [13,20]. This is justified because in our simulation setup, as described below, both species will be separated throughout, and no merging of colonies of different kinds will take place. In the (local) absence of one species, the dual-species models reduce to the single species models.

In this model formulation, all parameters are non-negative. See Table 1 for their definition and default values that will be used in the simulations. 
Table 1. Model parameters and their default values used in the simulations.

\begin{tabular}{l|l|l}
\hline Parameter & Symbol & Value \\
\hline Growth rate of species $i$ & $\mu_{i}$ & 1.0 \\
Cell lysis rate for species $i$ & $\delta_{i}$ & 0.1 \\
Substrate uptake rate of down-regulated cells of species & $\nu_{i}$ & $1.0 \times 10^{4}$ \\
Half saturation concentration for species $i$ & $\kappa_{i}$ & 0.1 \\
Factor of increased substrate uptake of up-regulated cells of species $i$ & $\sigma_{i}$ & 1.0 \\
Induction threshold for species $i$ & $\tau_{i}$ & 1.0 \\
Basal signal production rate & $\alpha$ & 4500 \\
Increased signal production rate of upregulated cells & $\beta$ & 45000 \\
Abiotic signal degradation rate & $\gamma$ & 0 \\
Dimerisation exponent for signal production & $n$ & 2.5 \\
\hline Diffusion coefficient of carbon substrate & $D_{C}$ & 33 \\
Diffusion coefficient of signal & $D_{S}$ & 16.5 \\
Biomass motility coefficient & $D_{M}$ & $1.0 \times 10^{-6}$ \\
Biomass diffusion nonlinearity exponent & $a$ & 4 \\
\hline Growth chamber length/width & $L$ & 0.5 \\
External substrate concentration & $C_{0}$ & 1.0 \\
\hline
\end{tabular}

\subsection{Physical Configuration and Initial and Boundary Conditions}

The simulation setup in our study is inspired by a micro-fluidic device that was introduced in [22] and is based on [23]. It consists of a rectangular growth chamber of extensions $2 L \times L \times H$, which is subdivided by a membrane into two sections of size $L \times L \times H$. This membrane is permeable to dissolved substrates $C$ and $S$, but not to bacteria. Along the lateral boundaries opposite of the internal membrane, both growth chambers are connected to tangential flow channels by like membranes. In the flow channels the substrate concentration is kept at a bulk concentration $C=C_{0}$, and the signal concentration is kept at nil. Thus, via diffusion across these external membranes, substrate is supplied to the growth channels, and signals are removed. The other boundaries of the growth chamber are impermeable to substrates. The height of the growth chamber $H$ is comparable to the length scale of bacteria, and $H \ll L$. This allows us to consider biofilm colonies as two-dimensional, spreading across the substratum but having constant height. The system is described then by a two-dimensional rectangular domain $\Omega=[0,2 L] \times[0, L]$. The boundary conditions are

$$
\begin{gathered}
\left.\partial_{n} M_{1,2}\right|_{\partial \Omega}=0, \\
\left.C\right|_{x_{1} \in\{0,2 L\}}=C_{0},\left.S\right|_{x_{1} \in\{0,2 L\}}=0,\left.\partial_{n} C\right|_{x_{2} \in\{0, L\}}=0,\left.\partial_{n} S\right|_{x_{2} \in\{0, L\}}=0 .
\end{gathered}
$$

Although the experimental device on which our simulation setup is based has a membrane separating both sections of the growth chamber, we do not specify internal boundary conditions. The purpose of the membrane is to allow the substrate and the signal, $C$ and $S$, to freely diffuse between both parts of the domain, but to prevent the colony bacteria from crossing over. Due to the porous medium degeneracy $D(0)=0$ in (6), initial data with compact support imply 
solutions with compact support. Thus a colony that initially is separated away from the inner boundary will at most reach it after some finite time $T$. Our simulations are always terminate before that occurs.

The initial conditions for the dissolved concentrations in our simulations are

$$
\left.S\right|_{t=0} \equiv 0,\left.\quad C\right|_{t=0}=C_{0} .
$$

For the biomass we introduce two subdomains $\Omega_{1}(0) \subset[0, L-\eta] \times[0, L] \subset \Omega$ and $\Omega_{2}(0) \subset[L+\eta, 2 L] \times[0, L] \subset \Omega, 0<\eta \ll L$, such that

$$
\left.M_{i}\right|_{t=0}=\left\{\begin{array}{ll}
m_{o i} \geq 0, & x \in \Omega_{i}(0), \\
0 & x \notin \Omega_{i}(0)
\end{array}, \quad i=1,2 .\right.
$$

Here $\Omega_{i}(0)$ consists of possibly several not connected compact regions that are very small compared to the domain $\Omega$. Mostly we will use these to be spherical or constructed from overlapping spherical regions.

\subsection{Numerical Implementation}

Besides the stiffness introduced by the disparity of time scales in the reaction terms, the model features two effects that make its numerical simulation challenging: the super-diffusion singularity as $M \rightarrow 1$ leads to blow-up of the diffusion coefficient, whereas the degeneracy at $M=0$ introduces a sharp interface that propagates with finite speed and along which the biomass gradient blows up. The model is first discretised in space, then in time, following the approach laid out in $[13,14]$ : For spatial discretisation a standard finite volume method is used on a uniform rectangular grid of size $2 m \times m$. Integrating (1) over each grid cell and using the Divergence Theorem gives

$$
\frac{d}{d t} \int_{v_{i, j}} M_{1} d x d y=\int_{\partial v_{i, j}} J_{n} d s+\int_{v_{i, j}} R_{1} d x d y, \quad i=1, \ldots, 2 m, j=1, \ldots, m,
$$

where $v_{i j}$ denotes the domain of the cell with grid index $(i, j), J_{n}:=D\left(M_{1}\right) \partial_{n} M_{1}$ denotes the outward normal flux across the grid cell boundary, and $R_{1}$ stands for the reaction terms in (1). To evaluate the area integrals in (11), we evaluate the dependent variables at the grid cell center,

$$
M_{1 ; i, j}(t):=M_{1}\left(t, x_{i}, y_{j}\right) \approx M_{1}(t,(i-1 / 2) \Delta x,(j-1 / 2) \Delta x)
$$

with $\Delta x=H / m$ and approximate the integral by the midpoint rule. The line integral in (11) is evaluated by considering every edge of the grid cell separately using the midpoint rule. To this end, the diffusion coefficient $D\left(M_{1}\right)$ in the midpoint of the cell edge is approximated by arithmetic averaging from the neighboring grid cell center points, and the derivative of $M_{1}$ across the cell edge by a central finite difference. It was shown previously that this approximates biofilm interface propagation well $[5,14]$. We get then for the biomass density in the grid cell center the ordinary differential equation

$$
\frac{d}{d t} M_{1 ; i, j}=\frac{1}{\Delta x}\left(J_{i+\frac{1}{2}, j}+J_{i-\frac{1}{2}, j}+J_{i, j+\frac{1}{2}}+J_{i, j-\frac{1}{2}}\right)+R_{1 ; i, j} .
$$


For the fluxes we have

$$
\begin{aligned}
& J_{i+\frac{1}{2}, j}= \begin{cases}\frac{1}{2 \Delta x}\left(D\left(M_{1 ; i+1, j}\right)+D\left(M_{1 ; i, j}\right)\right)\left(M_{1 ; i+1, j}-M_{1 ; i, j}\right) & \text { for } \quad i<2 m, \\
0 & \text { for } \quad i=2 m,\end{cases} \\
& J_{i-\frac{1}{2}, j}= \begin{cases}0 & \text { for } i=1, \\
\frac{1}{2 \Delta x}\left(D\left(M_{1 ; i, j}\right)+D\left(M_{1 ; i-1, j}\right)\right)\left(M_{1 ; i-1, j}-M_{1 ; i, j}\right) & \text { for } i>1,\end{cases} \\
& J_{i, j+\frac{1}{2}}= \begin{cases}\frac{1}{2 \Delta x}\left(D\left(M_{1 ; i, j+1}\right)+D\left(M_{1 ; i, j}\right)\right)\left(M_{1 ; i, j+1}-M_{1 ; i, j}\right) & \text { for } j<m, \\
0 & \text { for } j=m,\end{cases} \\
& J_{i, j-\frac{1}{2}}= \begin{cases}0 & \text { for } \quad j=1, \\
\frac{1}{2 \Delta x}\left(D\left(M_{1 ; i, j-1}\right)+D\left(M_{1 ; i, j}\right)\right)\left(M_{1 ; i, j-1}-M_{1 ; i, j}\right) & \text { for } \quad j>1 .\end{cases}
\end{aligned}
$$

The spatial discretization of the equations for $M_{2}, S, C$ follows the same principle, replacing the Neumann boundary conditions along the lateral boundaries by Dirichlet conditions for $S$ and $C$. After spatial discretization and lexiographical ordering we obtain a system of $8 \mathrm{~m}^{2}$ differential equations of the form

$$
\left\{\begin{array}{l}
\frac{d \mathbf{M}_{1}}{d t}=\mathcal{D}\left(\mathbf{M}_{1}\right) \mathbf{M}_{1}+\mathcal{R}_{1}(\mathbf{C}) \mathbf{M}_{1} \\
\frac{d \mathbf{M}_{2}}{d t}=\mathcal{D}\left(\mathbf{M}_{2}\right) \mathbf{M}_{2}+\mathcal{R}_{2}(\mathbf{C}) \mathbf{M}_{2} \\
\frac{d \mathbf{C}}{d t}=\mathcal{D}_{C} \mathbf{C}-\mathcal{R}_{1, C}(\mathbf{C}, \mathbf{S}) \mathbf{M}_{1}-\mathcal{R}_{2, C}(\mathbf{C}, \mathbf{S}) \mathbf{M}_{2}+\mathbf{b}_{C} \\
\frac{d \mathbf{S}}{d t}=\mathcal{D}_{S} \mathbf{S}+\mathcal{R}_{S}(\mathbf{C}, \mathbf{S}) \mathbf{M}_{1}-\gamma \mathcal{I} \mathbf{S}+\mathbf{b}_{S}
\end{array}\right.
$$

where vectors $\mathbf{M}_{1}, \mathbf{M}_{2}, \mathbf{C}, \mathbf{S}$ contain the grid approximations of the dependent variables, $\mathcal{I}$ is the identity matrix in $\mathbb{R}^{2 m^{2} \times 2 m^{2}}$. The matrices $\mathcal{D}$ and $\mathcal{D}_{N, S}$ contain the spatial derivative terms. They are symmetric, and weakly diagonally dominant with non-positive main diagonals and non-negative off-diagonals. The matrices $\mathcal{R}_{1}, \mathcal{R}_{2}, \mathcal{R}_{1, C}, \mathcal{R}_{2, C}, \mathcal{R}_{S}$ are diagonal and contain the reaction terms; vectors $\mathbf{b}_{C S}$ contain contributions of the Dirichlet boundary conditions.

The semi-discrete system (13) is a sparse lattice ODE that satisfies a Lipschitz condition and preserves non-negativity. We use the embedded RosenbrockWanner Method ROS3PRL [21]. The Jacobian linear systems are solved using a Jacobi-preconditioned BiCGSTAB method. Most of the code was implemented in-house using Fortran (with Intel, GNU, and Portland Group compilers) and prepared for execution on shared memory computers using OpenMP. For the BiCGSTAB algorithm we used an OpenMP parallelised version of the implementation in Sparskit [19,25]. This uses reverse communication and external inner products and sparse matrix vector products. Both the diffusion operators of the model and the Jacobian matrices are organised in sparse diagonal format. Simulations reported here have been carried out on Intel Xeon based Lenovo P520 and P710 workstations, and on two heterogeneous clusters of the Compute Canada network (SHARCNET's Graham, and WestGrid's cedar). ParaView was used for visual postprocessing. 


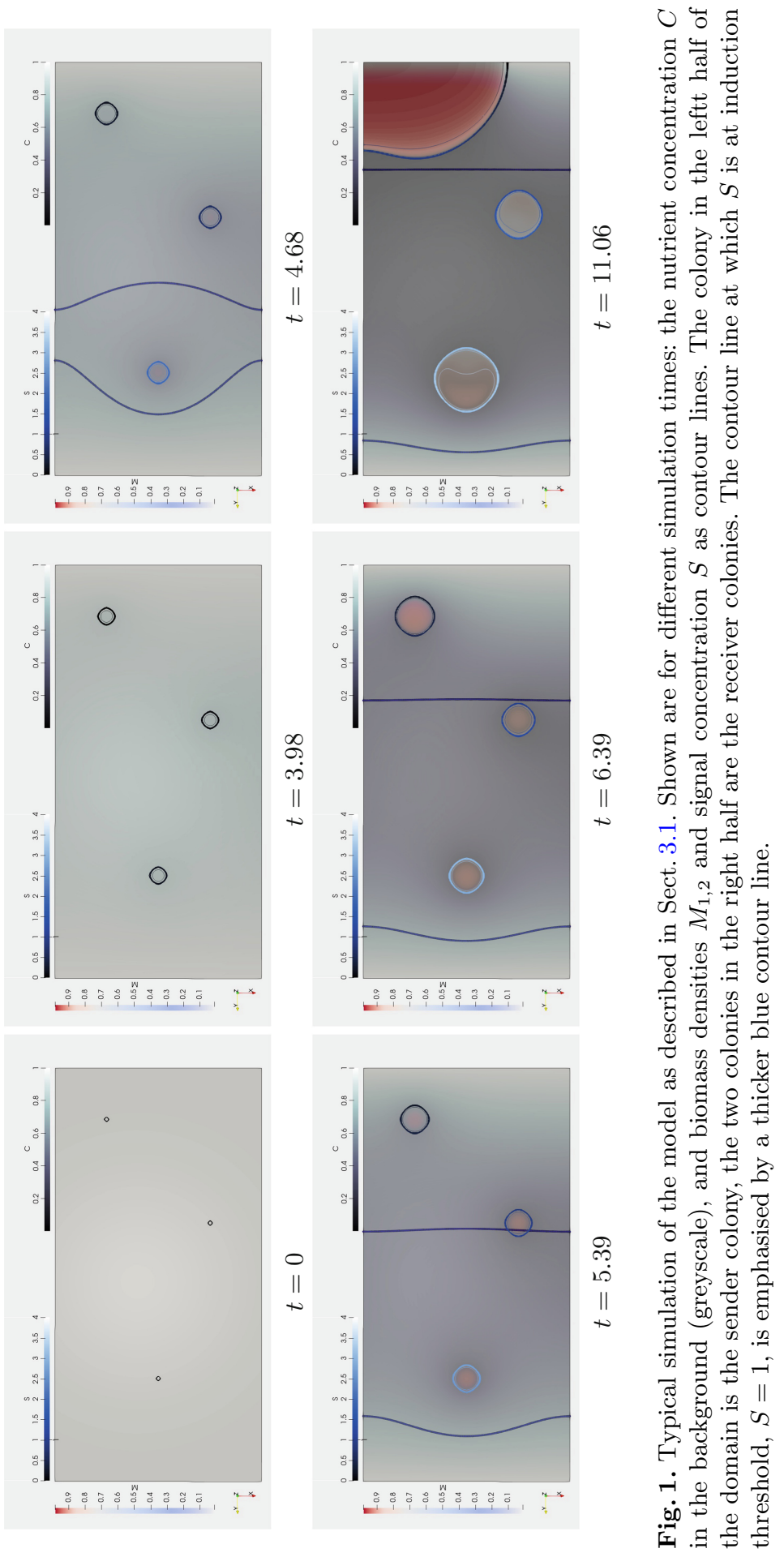




\section{Results}

\subsection{An Illustrative Simulation}

For illustration, we simulate a system with a small sender colony in the center of the right half of the domain, and two receiver colonies in the left half, one is closer to the boundary through which nutrients are supplied and autoinducers removed, one closer to the center line. The results are shown in Fig. 1. Initially, the biomass in all colonies increases; they start expanding at $t \approx 1$. As the colonies grow the substrate concentration there decreases. This induces steeper nutrient gradients at the boundaries and, thus, increases mass transfer into the system, promoting growth of all colonies. The close a colony is to a lateral boundary, the faster it expands. This becomes especially pronounced when the nutrient concentration becomes small, at around $t \approx 6$. The increased substrate uptake by induced cells leads to a local minimum in the sender colony. The signal remains below induction threshold $S<1$, everywhere until $t \approx 4.5$. Once upregulation starts, it is rapid in the sender colony. The signal concentration is always highest there. From here it diffuses into the domain. Much of the signal is removed through the lateral boundary behind the sender colony, but a considerable amount is transported into the other half of the domain, toward the receiver colonies. At $t=5.39$ the signal at induction threshold levels the smaller receiver colony, which is quickly encompassed in the region of up-regulation $S>1$. As it upregulates, its nutrient consumption increases, slowing down growth and expansion. At $t=$ 6.39 this colony is entirely contained in the region of upregulation. As signal is continuously removed from the environment, it never exceeds a maximum value of $S \approx 4.2$, which is attained in the sender colony.

This simulation was carried out on a grid of size $400 \times 200$. To validate parallel performance of the code, a speed-up test was performed for the time interval $3.90<t<4.69$, which encompasses the onset of upregulation. This was carried out on a Lenovo P520 worksation with an Intel Xeon E5-1660 processor, and code compiled using the Intel fortran compiler. Vis-a-vis sequential execution, we found acceleration by factor 1.85 on 2 cores and 3.05 on 4 cores. Further speedup beyond this was found to be insignificant for a problem of this size (e.g. 3.46 on 8 cores). We repeated this with the gfortran and the portland group complers with similar results. This finding is in good agreement with earlier results for similar problems with the same or different (namely a Jacobian free but not error controlled) time integration strategies $[13,14,19]$.

\subsection{Simulation Experiment: Effect of Cross-Talk on Sender Growth}

To investigate the effect that cross-talk activity has on the biofilm community, we compare the results of (i) the default setup described in the previous section with two variants: (ii) a system with the sender colony only, $m_{o 2}=0$; (iii) a system where the receiver does not respond to the signal, $\sigma_{2}=0$. This allows us to study how in our system cross-talk affects the signal producer. 


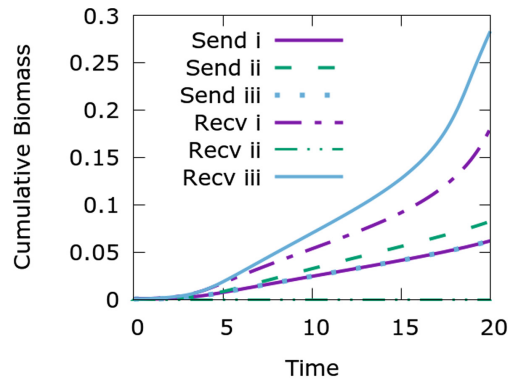

(a)

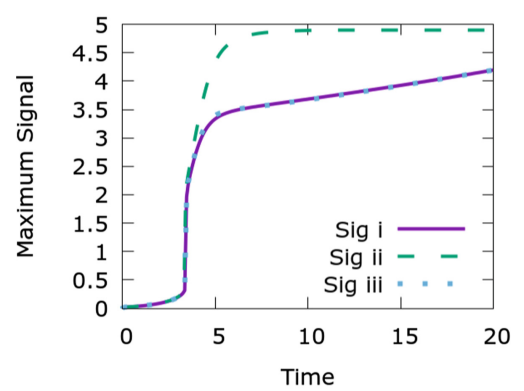

(c)

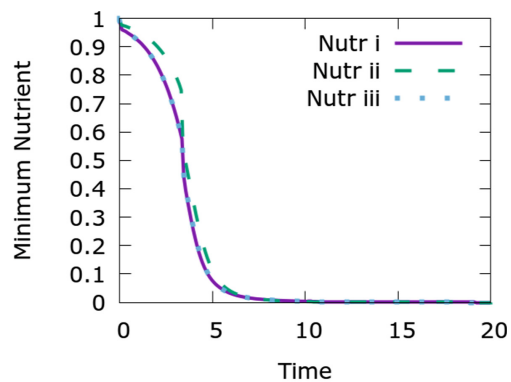

(b)

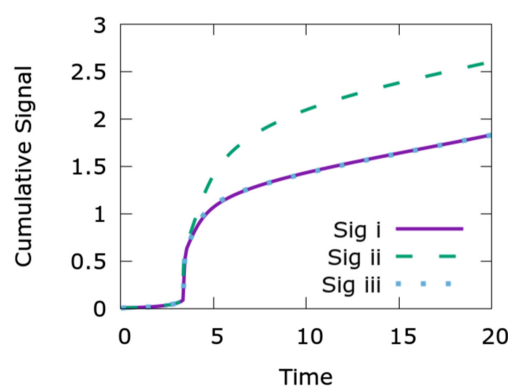

(d)

Fig. 2. Total biomass, maximum signal concentration, total amount of signal, and minimum substrate concentration in simulations of cases (i)-(iii) of Sect. 3.2.

We report in Fig. 2 the following quantities of interest, which are all functions of time: total mass of sender and receiver species, minimum substrate concentration in the system, maximum signal concentration, and total amount of signal. In the absence of receiver colonies, case (ii), the nutrient availability for senders increases due to a lack of competition. This leads to a faster growth of senders, thus more signals are produced. Also the minimum nutrient concentration remains slightly above the case with three colonies present. If the receivers do not respond to the signal, case (iii), they do not increase nutrient uptake when the signal concentration enters a range of induction, $S>1$. Thus the available nutrients are more efficiently converted into new biomass, leading to a growth advantage vis-a-vis the base line scenario of case (i). This increased growth of receivers does not translate into a reduction in nutrients available to senders, and thus the signal concentrations in case (iii) is almost identical to case (i).

\subsection{Simulation Experiment: The Role of Signal Degradation}

The model contains in (4) abiotic signal degradation at rate $\gamma$. In the simulations in Sects. 3.1, 3.2 this term was turned off, $\gamma=0$. This parameter will be key in exploring in future studies quorum quenching strategies in cross-talk systems, i.e. attempts to suppress quorum sensing by lowering signals, which often have 

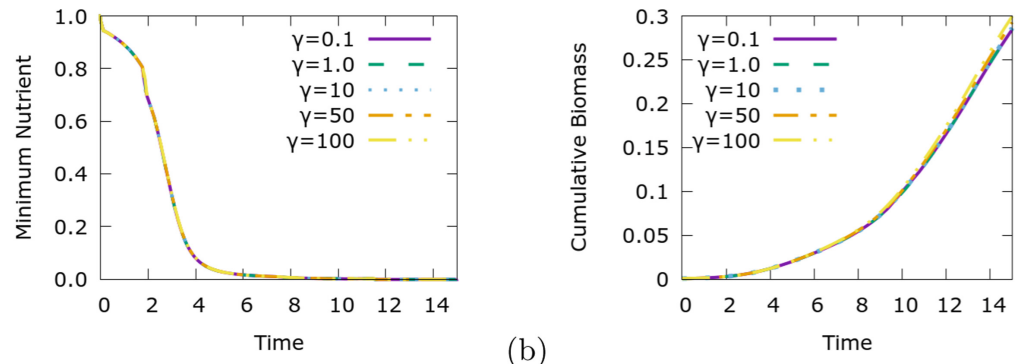

(a)

(b)
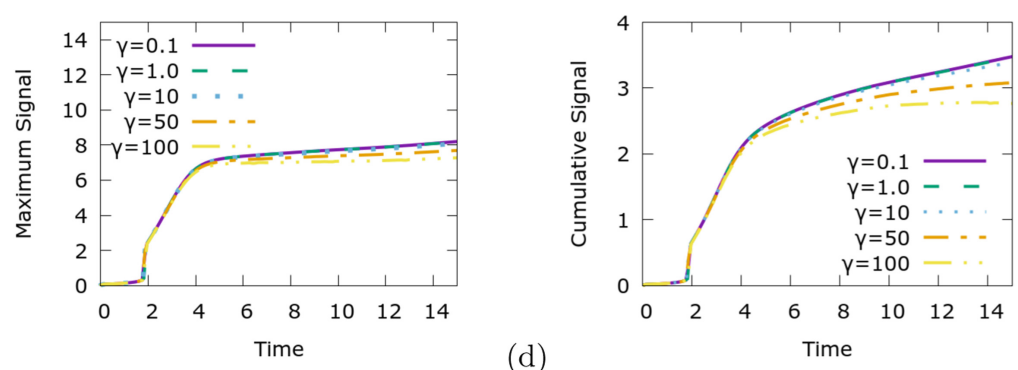

Fig. 3. Minimum substrate concentration, maximum signal concentration and total amount of signal in simulations with different signal degradation rates $\gamma$ and $D_{S}=8$, cf. Sect. 3.3. No effect of $\gamma$ on the sender population size was observed.

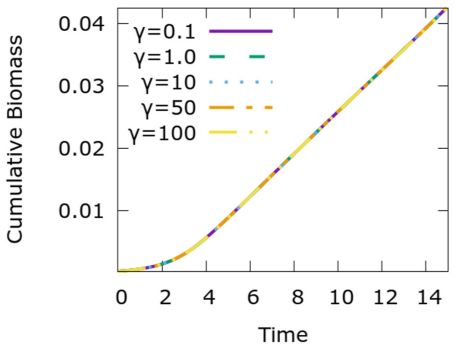

(a)

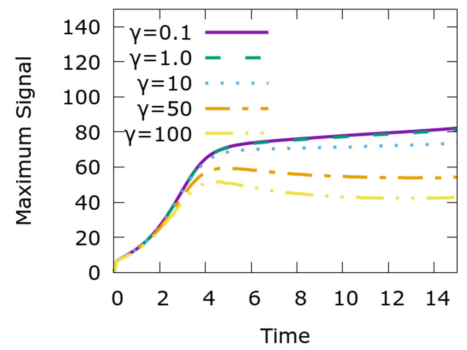

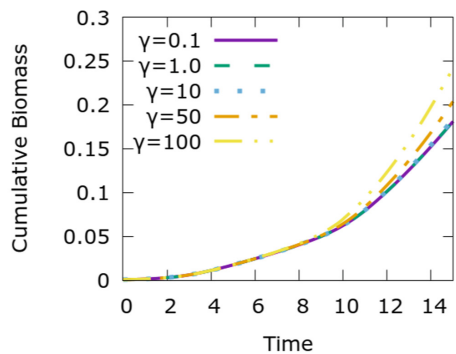

(b)

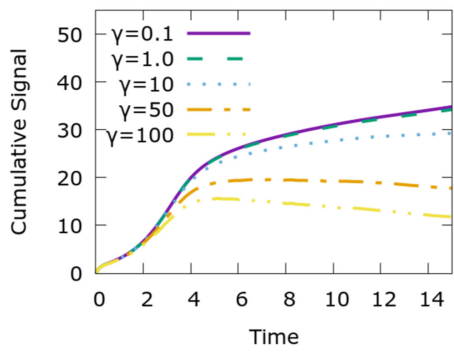

(d)

Fig. 4. Total biomass, maximum signal concentration and total amount of signal in simulations with varying signal degradation rates $\gamma$, and $D_{S}=0.8$, cf. Sect. 3.3. No effect of $\gamma$ on the minimum substrate concentration was observed. 
been suggested as adjuvant strategies to antibiotics in the treatment of biofilm borne infections [9]. Increasing $\gamma$ will lower the signal concentration and thus delay (or even prevent) upregulation, which leads in our formulation to an increased substrate concentration, thus to faster biomass growth, hence more signal being produced, etc. The net effect of these processes pulling in opposite direction is difficult to predict, and likely depends on the actual parameter values.

We vary $\gamma$ in the range $\gamma=0.1 \sim 100$. The signal diffusion coefficient was set to $D_{S}=8$. In Fig. 3 we present the same quantities of interests that we have reported in Sect. 3.2. Differences in signal concentration as $\gamma$ varies become noticeable only after the onset of induction, at $t \approx 3$. However, differences in signal concentration in a range clearly above induction threshold, $S>\tau$, have only little effect, since there $h(S, \tau) \approx 1$. Accordingly, in our simulations, $\gamma$ has almost now influence on the population and cross-talk dynamics.

To explore this further, we carried out additional simulations. Although the diffusion coefficients are difficult to control experimentally, we re-ran the simulation with $D_{S}=80$, a value that is likely too large to be practically relevant. Increased diffusion implies a faster removal of autoinducer signals. Also in these simulations one could observe the effect of $\gamma$ on the signal concentration, but due to their fast washout, the system was never induced, such that $h(S, \tau) \approx 0$. Therefore, also in this regime signal degradation did not affect population and cross-talk behaviour (data not shown). On the other hand, for a substantially lower signal diffusion concentration, $D_{S}=0.8$ (too low to be practically relevant), autoinducers are not removed fast enough and can accumulate, cf Fig. 4. Due to the slow transport in the system, reactions dominates over diffusion, so that the signal concentration in the receiver region remains small, making more substrate available for growth of receiver population. This suggests that the efficacy of signal degradation depends strongly on the mass transport conditions in the system, which often are not easy to control, e.g. in a medical context.

\section{Conclusion}

In the course of this study the following lessons were learned:

1. The dynamics and efficacy of bacterial cross-talk, like quorum sensing itself, is greatly affected by transport processes in the environment and not a mere consequence of population size. Such transport effects can introduce spatial heterogeneity in population behaviour. It is conceivable to have in one biofilm community receiver colonies of the same species that are entirely induced, and others that remain down-regulated.

2. In our simulations the only effect of upregulation was an increased consumption of nutrients. We did not track explicitly virulence factors into which these additional resources are invested. In more specific applications, accounting for the effect of up-regulation in more detail, the question will arise whether the purpose of cross-talk between species is to benefit the sender over the recipient or vice versa, or whether the relationship is symbiotic. This will also be a question about the nature of the underlying autoinduction system, which will 
possibly introduce additional positive or negative feedback. Further investigation will be warranted, requiring a model extension and to account for additional Fickian or degenerate diffusion-reaction equations, thus increasing computational complexity.

3. When developing quorum quenching strategies to control cross-talk it will be paramount that those need to take into account also physical conditions of the system, in particular as they pertain to signal transport, and not only biological and biochemical aspects.

4. The simulation setup that we introduced here was inspired by a recently proposed experimental biofilm growth reactor. It allows us to reduce the influence of physical unrealistic boundary condition effects, to which most multi-dimensional biofilm simulation studies are exposed. Moreover, it allows to use a $2 \mathrm{D}$ description as a natural setting for such simulations. This seems a promissing setup for further multi-dimensional biofilm simulation studies going forward.

Acknowledgement. We thank WestGrid, SHARCNET and Compute Canada for access to compute resources used for some simulations that we reported here.

\section{References}

1. Barr, H.L., Halliday, N., Cámara, M., Barrett, D.A., Williams, P., Forrester, D.L., et al.: Pseudomonas aeruginosa quorum sensing molecules correlate with clinical status in cystic fibrosis. Eur. Respir. J. 46, 1046-1054 (2015)

2. Davies, D.G.: The involvement of cell-to-cell signals in the development of a bacterial biofilm. Science 280, 295-298 (1998)

3. Doberva, M., Stien, D., Sorres, J., Hue, N., Sanchez-Ferandin, S., Eparvier, V., et al.: Large diversity and original structures of acyl-homoserine lactones in strain MOLA 401, a marine rhodobacteraceae bacterium. Front. Microbiol. 8, 1152 (2017)

4. Dulla, G.F.J., Lindow, S.E.: Acyl-homoserine lactone-mediated cross talk among epiphytic bacteria modulates behavior of Pseudomonas syringae on leaves. ISME J. 3, 825-834 (2009)

5. Eberl, H.J., Demaret, L.: A finite difference scheme for a degenerated diffusion equation arising in microbial ecology. Electron. J. Differ. Equ. 15, 77-95 (2007)

6. Eberl, H.J., Parker, D.F., van Loosdrecht, M.C.M.: A new deterministic spatiotemporal continuum model for biofilm development. J. Theor. Med. 3(3), 161-175 (2001)

7. Emerenini, B., Hense, B.A., Kuttler, C., Eberl, H.J.: A mathematical model of quorum sensing induced biofilm detachment. PlosOne 10(7), e0132385 (2015)

8. Emerenini, B.O., Sonner, S., Eberl, H.J.: Mathematical analysis of a quorum sensing induced biofilm dispersal model. Math. Biosc. Eng. 14(3), 625-653 (2017)

9. Fong, J., Zhang, C., Yang, R., Boo, Z.Z., Tan, S.K., Nielsen, T.E., et al.: Combination therapy strategy of quorum quenching enzyme and quorum sensing inhibitor in suppressing multiple quorum sensing pathways of P. aeruginosa. Sci. Rep. 8(1), 1155 (2018)

10. Frederick, M.R., Kuttler, C., Hense, B.A., Müller, J., Eberl, H.J.: A mathematical model of quorum sensing in patchy biofilm communities with slow background flow. Can. Appl. Math. Quart. 18(3), 267-298 (2010) 
11. Frederick, M.R., Kuttler, C., Hense, B.A., Eberl, H.J.: A mathematical model of quorum sensing regulated EPS production in biofilms. Theor. Biol. Med. Model. 8, 8 (2011)

12. Fuqua, W., Winans, S., Greenberg, E.: Quorum sensing in bacteria: the LuxRLuxI family of cell density-responsive transcriptional regulators. J. Bacteriol. 176, 269-275 (1994)

13. Ghasemi, M., Eberl, H.J.: Extension of a regularization based time-adaptive numerical method for a degenerate diffusion-reaction biofilm growth model to systems involving quorum sensing. Procedia Comput. Sci. 108, 1893-1902 (2017)

14. Ghasemi, M., Eberl, H.J.: Time adaptive numerical solution of a highly degenerate diffusion-reaction biofilm model based on regularisation. J. Sci. Comput. 74, 10601090 (2018)

15. Ghasemi, M., Hense, B.A., Kuttler, C., Eberl, H.J.: Simulation based exploration of quorum sensing triggered resistance of biofilms to antibiotics. Bull. Math. Biol. 80(7), 1736-1775 (2018)

16. Hense, B.A., Kuttler, C., Mïler, J., Rothballer, M., Hartmann, A., Kreft, J.-U.: Does efficiency sensing unify diffusion and quorum sensing? Nat. Rev. Microbiol. 5, 230-239 (2007)

17. Kim, M.K., Ingremeau, F., Zhao, A., Bassler, B.L., Stone, H.A.: Local and global consequences of flow on bacterial quorum sensing. Nat. Microbiol. 1, 15005 (2016)

18. Lewandowski, Z., Beyenal, H.: Fundamentals of Biofilm Research. CRC Press, Boca Raton (2007)

19. Muhammad, N., Eberl, H.J.: OpenMP parallelization of a mickens time-integration scheme for a mixed-culture biofilm model and its performance on multi-core and multi-processor computers. In: Mewhort, D.J.K., Cann, N.M., Slater, G.W., Naughton, T.J. (eds.) HPCS 2009. LNCS, vol. 5976, pp. 180-195. Springer, Heidelberg (2010). https://doi.org/10.1007/978-3-642-12659-8_14

20. Rahman, K.A., Sudarsan, R., Eberl, H.J.: A mixed culture biofilm model with cross-diffusion. Bull. Math. Biol. 77(11), 2086-2124 (2015)

21. Rang, J.: Improved traditional Rosenbrock-Wanner methods for stiff ODEs and DAEs. J. Comput. Appl. Math. 286, 128-144 (2015)

22. Shankles, P.G.: Interfacing to biological systems using microfluidics. Ph.D. dissertation, University of Tennessee, Knoxville (2018)

23. Shankles, P.G., Timm, A.C., Doktycz, M.J., Retterer, S.T.: Fabrication of nanoporous membranes for tuning microbial interactions and biochemical reactions. J. Vac. Sci. Tech. B. 33(6), 06FM031-06M031-8 (2015)

24. Silva, K.P.T., Chellamuthu, P., Boedicker, J.Q.: Quantifying the strength of quorum sensing crosstalk within microbial communities. PLoS Comput. Biol. 13(1), e1005809 (2017)

25. Saad, Y.: SPARSKIT: a basic tool for sparse matrix computations (1994). http:// www.users.cs.umn.edu/saad/software/SPARSKIT/sparskit.htm

26. Sonner, S., Efendiev, M.A., Eberl, H.J.: On the well-posedness of a mathematical model of quorum-sensing in patchy biofilm communities. Math. Meth. Appl. Sci. 34(13), 1667-1684 (2011)

27. Stewart, P.S., Costerton, J.E.: Antibiotic resistance of bacteria in biofilms. Lancet 358, 135-38 (2001) 